\title{
A Bijection on Dyck Paths and its Cycle Structure
}

\author{
DAVID CALLAN \\ Department of Statistics \\ University of Wisconsin-Madison \\ 1300 University Ave \\ Madison, WI 53706-1532 \\ callan@stat.wisc.edu
}

Submitted: Nov 29, 2006; Accepted: Mar 23, 2007; Published: Apr 4, 2007

Mathematics Subject Classification: 05A15, 05A19

\begin{abstract}
The known bijections on Dyck paths are either involutions or have notoriously intractable cycle structure. Here we present a size-preserving bijection on Dyck paths whose cycle structure is amenable to complete analysis. In particular, each cycle has length a power of 2 . A new manifestation of the Catalan numbers as labeled forests crops up en route as does the Pascal matrix mod 2. We use the bijection to show the equivalence of two known manifestations of the Motzkin numbers. Finally, we consider some statistics on the new Catalan manifestation and the identities they interpret.
\end{abstract}

\section{Introduction}

There are several bijections on Dyck paths in the literature $[1,2,3,4,5,6,7,8,9$, 10, 11], usually introduced to show the equidistribution of statistics: if a bijection sends statistic A to statistic B, then clearly both have the same distribution. Another aspect of such a bijection is its cycle structure considered as a permutation on Dyck paths. Apart from involutions, this question is usually intractable. For example, Donaghey [7] introduces a bijection, obtains some results on a restriction version, and notes its apparently chaotic behavior in general. In similar vein, Knuth [8] defines a conjugate $(R)$ and transpose $(T)$, both involutions, on ordered forests, equivalently on Dyck paths, and asks when they commute [8, Ex. 17, 7.2.1.6], equivalently, what are the fixed points of $(R T)^{2}$ ? This question is still open. (Donaghey's bijection is equivalent to the composition $R T$. 
In this paper, after reviewing Dyck path terminology $(\S 2)$, we recursively define a new bijection $F$ on Dyck paths $(\S 3)$, analyze its cycle structure $(\S 4-\S 8)$, and present some applications $(\S 9-\S 10)$. Section 4 treats the restriction of $F$ to primitive paths that avoid the subpath $D U U$, and involves an encounter with the Pascal matrix mod 2. Section 5 deals with arbitrary primitive paths. Section 6 introduces so-called LCO forests, a Catalan manifestation that permits an explicit description of $F$ and its orbits. We show that each orbit has size (length) a power of 2 and characterize orbits of given size in terms of subpath avoidance. In particular, the fixed points of $F$ are those Dyck paths that avoid $D U D D$ and $U U P^{+} D D$ where $P^{+}$denotes a nonempty Dyck path. Section 7 finds generating functions and explains a divisibility property of the orbit sizes. Section 8 uses the bijection $F$ to show the equivalence of two known manifestations of the Motzkin numbers. Finally, Section 9 considers some statistics on LCO forests and the identities they interpret.

\section{Dyck Path Terminology}

A Dyck path, as usual, is a lattice path of upsteps $U=(1,1)$ and downsteps $D=$ $(1,-1)$, the same number of each, that stays weakly above the horizontal line, called ground level, that joins its initial and terminal points (vertices). A peak is an occurrence of $U D$, a valley is a $D U$.



A Dyck 7-path with 2 components, $2 D U D$ s, and height 3

The size (or semilength) of a Dyck path is its number of upsteps and a Dyck path of size $n$ is a Dyck $n$-path. The empty Dyck path (of size 0) is denoted $\epsilon$. The number of Dyck $n$-paths is the Catalan number $C_{n}$, sequence A000108 in OEIS. The height of a vertex in a Dyck path is its vertical height above ground level and the height of the path is the maximum height of its vertices. A return downstep is one that returns the path to ground level. A primitive Dyck path is one with exactly one return (necessarily at the end). Note that the empty Dyck path $\epsilon$ is not primitive. The returns of a nonempty Dyck path split it into one or more primitive Dyck paths, called its components. Upsteps and downsteps come in matching pairs: travel due east from an upstep to the first downstep encountered. More precisely, $D_{0}$ is the matching downstep for upstep $U_{0}$ if $D_{0}$ terminates the shortest Dyck subpath that starts with $U_{0}$. We use $\mathcal{P}$ to denote the set of primitive Dyck paths, $\mathcal{P}_{n}$ for $n$-paths, $\mathcal{P}(D U U)$ for the subset that avoid $D U U$ as a subpath, and $\mathcal{P}[D U U]$ for the subset that contain at least one $D U U$. A path $U U U D U D D D$, for example, is abbreviated $U^{3} D U D^{3}$. 


\section{The Bijection $F$}

Define a size-preserving bijection $F$ on Dyck paths recursively as follows. First, $F(\epsilon)=$ $\epsilon$ and for a non-primitive Dyck path $P$ with components $P_{1}, P_{2}, \ldots, P_{r}(r \geq 2), F(P)=$ $F\left(P_{1}\right) F\left(P_{2}\right) \ldots F\left(P_{r}\right)$ (concatenation). This reduces matters to primitive paths. From a consideration of the last vertex at height 3 (if any), every primitive Dyck path $P$ has the form $U Q(U D)^{i} D$ with $i \geq 0$ and $Q$ a Dyck path that is either empty (in case no vertex is at height 3 ) or ends with $D D$; define $F(P)$ by

$$
F(P)= \begin{cases}U^{i+1} F(R) U D D^{i+1} & \text { if } Q \text { is primitive, say } Q=U R D, \text { and } \\ U^{i+1} F(Q) D^{i+1} & \text { if } Q \text { is not primitive. }\end{cases}
$$

Schematically,

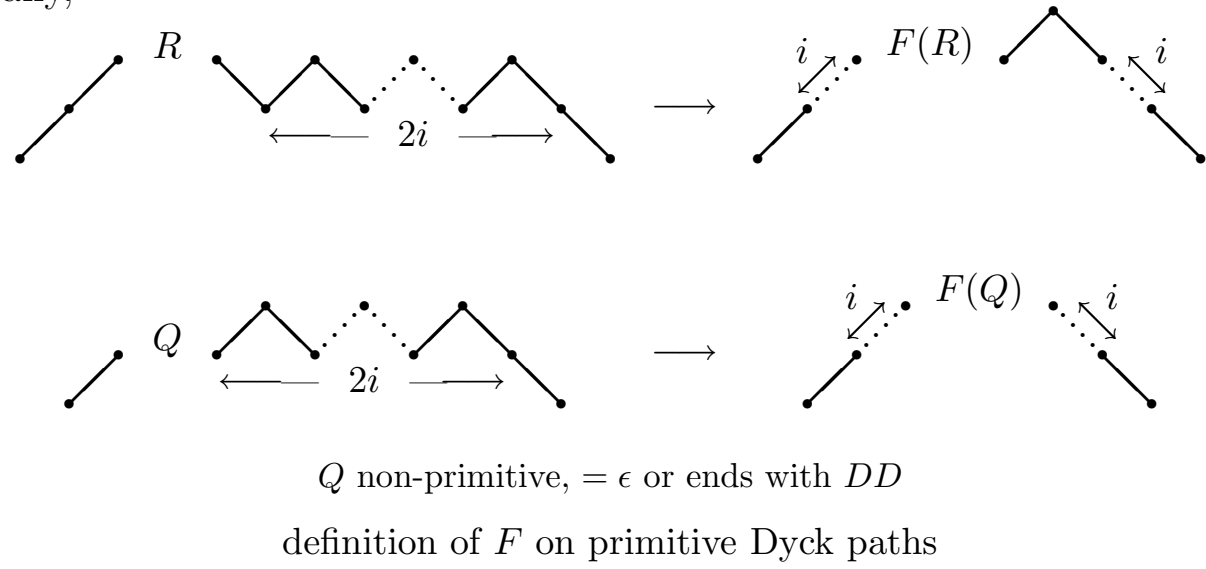

Note that $R=\epsilon$ in the top left path duplicates a case of the bottom left path but no matter: both formulas give the same result.

The map $G$, defined as follows, serves as an inverse of $F$ and hence $F$ is indeed a bijection. Again, $G(\epsilon)=\epsilon$ and for a non-primitive Dyck path $P$ with components $P_{1}, P_{2}, \ldots, P_{r}(r \geq 2), G(P)=G\left(P_{1}\right) G\left(P_{2}\right) \ldots G\left(P_{r}\right)$. By considering the lowest valley vertex, every primitive Dyck path has the form $U^{i+1} Q D^{i+1}$ with $i \geq 0$ and $Q$ a nonprimitive Dyck path $(Q=\epsilon$ in case valley vertices are absent); define $G(P)$ by

$$
G(P)= \begin{cases}U U G(R) D(U D)^{i} D & \text { if } Q \text { ends with } U D, \text { say } Q=R U D, \text { and } \\ U G(Q)(U D)^{i} D & \text { otherwise. }\end{cases}
$$

The bijection $F$ is the identity on Dyck paths of size $\leq 3$, except that it interchanges $U^{3} D^{3}$ and $U^{2} D U D^{2}$. Its action on primitive Dyck 4-paths is given in the Figure below. 
Dyck path $P \quad \longrightarrow \quad$ image $F(P)$


action of $F$ on primitive Dyck 4-paths

\section{$4 F$ on Primitive $D U U$-avoiding Paths}

To analyze the structure of $F$ a key property, clear by induction, is that it preserves \# DUUs, in particular, it preserves the property "path avoids $D U U$ ". A $D U U$-avoiding Dyck $n$-path corresponds to a composition $\mathbf{c}=\left(c_{1}, c_{2}, \ldots, c_{h}\right)$ of $n$ via $c_{i}=$ number of $D \mathrm{~s}$ ending at height $h-i, i=1,2, \ldots, h$ where $h$ is the height of the path:

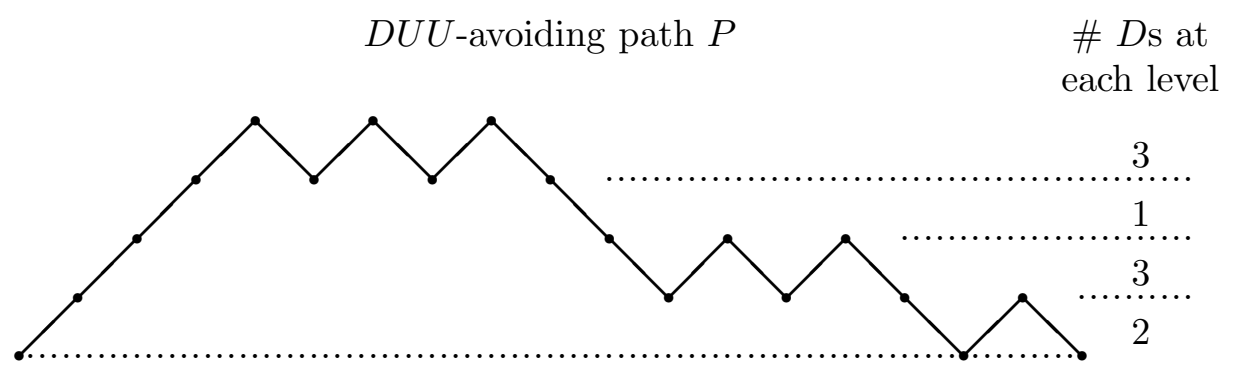

$$
\text { DUU-avoiding path } P \quad \leftrightarrow \quad \text { composition }(3,1,3,2)
$$

Under this correspondence, $F$ acts on compositions of $n: F$ is the identity on com- 
positions of length 1 , and for $\mathbf{c}=\left(c_{i}\right)_{i=1}^{r}$ with $r \geq 2, F(\mathbf{c})$ is the concatenation of IncrementLast $\left(F\left(c_{1}, \ldots, c_{r-2}\right)\right), 1^{c_{r-1}-1}, c_{r}$ where Increment Last means "add 1 to the last entry" and the superscript refers to repetition. In fact, $F$ can be described explicitly on compositions of $n$ (increment means "increase by 1 ") :

Proposition 1. For a composition $\mathbf{c}$ of $n, F(\mathbf{c})$ is given by the following algorithm. For each entry $c$ in even position measured from the end (so the last entry is in position 1), replace it by $c-11 s$ and increment its left neighbor.

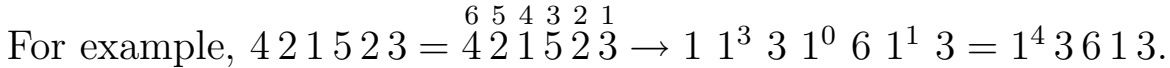

Primitive $D U U$-avoiding Dyck $n$-paths correspond to compositions of $n$ that end with a 1 . Let $\mathcal{C}_{n}$ denote the set of such compositions. Thus $\left|\mathcal{C}_{1}\right|=1$ and for $n \geq 2,\left|\mathcal{C}_{n}\right|=2^{n-2}$ since there are $2^{n-2}$ compositions of $n-1$.

Denote the length of a composition $\mathbf{c}$ by \#c. The size of $\mathbf{c}$ is the sum of its entries. The parity of $\mathbf{c}$ is the parity (even/odd) of \#c. There are two operations on nonempty compositions that increment the size: $\mathrm{P}=$ prepend 1 , and $\mathrm{I}=$ increment first entry. For example, for $\mathbf{c}=(4,1,1)$ we have $\operatorname{size}(\mathbf{c})=6, \quad \# \mathbf{c}=3$, the parity of $\mathbf{c}$ is odd, $\mathrm{P}(\mathbf{c})=(1,4,1,1), \mathrm{I}(\mathbf{c})=(5,1,1)$.

Lemma 2. $\mathrm{P}$ changes the parity of a composition while I preserves it.

We will call $\mathrm{P}$ and I augmentation operators on $\mathcal{C}_{n}$ and for $\mathrm{A}$ an augmentation operator, $A^{\prime}$ denotes the other one.

Lemma 3. Let $\mathrm{A}$ be an augmentation operator. On a composition $\mathbf{c}$ with $\# \mathbf{c} \geq 2$, $\mathrm{A} \circ F=F \circ \mathrm{A}$ if $\# \mathbf{c}$ is odd and $\mathrm{A} \circ F=F \circ \mathrm{A}^{\prime}$ if $\# \mathbf{c}$ is even.

This follows from Proposition 1.

Using Lemma 3 to determine $A_{i}$ for $i \geq 2$, an $F$-orbit $\left(\mathbf{c}_{1}, \ldots, \mathbf{c}_{m}\right)$ in $\mathcal{C}_{n}$ together with an augmentation operator $A_{1} \in\{P, I\}$ yields part of an $F$-orbit in $\mathcal{C}_{n+1}$ via a "commutative diagram" as shown:

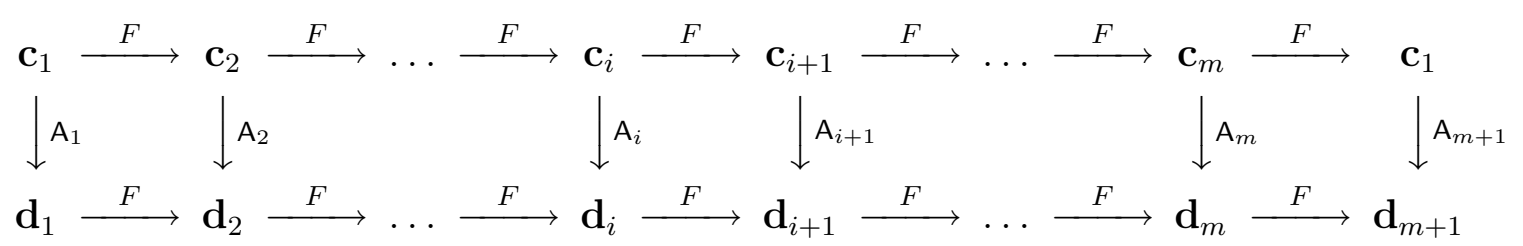

Let $B\left(\mathbf{c}_{1}, \mathrm{~A}_{1}\right)$ denote the sequence of compositions $\left(\mathbf{d}_{1}, \ldots, \mathbf{d}_{m}\right)$ thus produced. By Lemma $3, \mathrm{~A}_{i+1}=\mathrm{A}_{i}$ or $\mathrm{A}_{i}^{\prime}$ according as $\# \mathbf{c}_{i}$ is odd or even $(1 \leq i \leq m)$. Hence, if the orbit of $\mathbf{c}_{1}$ contains an even number of compositions of even parity, then $A_{m+1}=A_{1}$ and so $\mathbf{d}_{m+1}=\mathbf{d}_{1}$ and $B\left(\mathbf{c}_{1}, \mathrm{~A}_{1}\right)$ is a complete $F$-orbit in $\mathcal{C}_{n+1}$ for each of $\mathrm{A}_{1}=\mathrm{P}$ and $A_{1}=$ I. On the other hand, if the orbit of $\mathbf{c}_{1}$ contains an odd number of compositions of even parity, then $A_{m+1}=A_{1}^{\prime}$ and the commutative diagram will extend for another $m$ squares before completing an orbit in $\mathcal{C}_{n+1}$, consisting of the concatenation of $B\left(\mathbf{c}_{1}, \mathrm{P}\right)$ 
and $B\left(\mathbf{c}_{1}, \mathbf{I}\right)$, denoted $B\left(\mathbf{c}_{1}, \mathbf{P}, \mathbf{I}\right)$. In the former case orbit size is preserved; in the latter it is doubled.

Our goal here is to generate $F$-orbits recursively and to get induction going, we now need to investigate the parities of the compositions comprising these "bumped-up" orbits $B(\mathbf{c}, \mathrm{A})$ and $B(\mathbf{c}, \mathrm{P}, \mathrm{I})$. A bit sequence is a sequence of $0 \mathrm{~s}$ and $1 \mathrm{~s}$. In the sequel all operations on bit sequences are modulo 2 . Let $S$ denote the partial sum operator on bit sequences: $\mathbf{S}\left(\left(\epsilon_{1}, \epsilon_{2}, \ldots, \epsilon_{m}\right)\right)=\left(\epsilon_{1}, \epsilon_{1}+\epsilon_{2}, \ldots, \epsilon_{1}+\epsilon_{2}+\ldots+\epsilon_{m}\right)$. Let $\mathbf{e}_{m}$ denote the all $1 \mathrm{~s}$ bit sequence of length $m$ and let e denote the infinite sequences of $1 \mathrm{~s}$. Thus $\mathbf{S e}=(1,0,1,0,1, \ldots)$. Let $\mathbf{P}$ denote the infinite matrix whose $i$ th row $(i \geq 0)$ is $\mathbf{S}^{i} \mathbf{e}\left(\mathbf{S}^{i}\right.$ denotes the $i$-fold composition of $\mathbf{S})$. The $(i, j)$ entry $p_{i j}$ of $\mathbf{P}$ satisfies $p_{i j}=p_{i-1, j}+p_{i, j-1}$ and hence $\mathbf{P}$ is the symmetric Pascal matrix $\bmod 2$ with $(i, j)$ entry $=\left(\begin{array}{c}i+j \\ i\end{array}\right) \bmod 2$.

$$
\mathbf{P}=\left(\begin{array}{ccccccccc}
1 & 1 & 1 & 1 & 1 & 1 & 1 & 1 & \ldots \\
1 & 0 & 1 & 0 & 1 & 0 & 1 & 0 & \\
1 & 1 & 0 & 0 & 1 & 1 & 0 & 0 & \\
1 & 0 & 0 & 0 & 1 & 0 & 0 & 0 & \\
1 & 1 & 1 & 1 & 0 & 0 & 0 & 0 & \\
1 & 0 & 1 & 0 & 0 & 0 & 0 & 0 & \\
1 & 1 & 0 & 0 & 0 & 0 & 0 & 0 & \\
1 & 0 & 0 & 0 & 0 & 0 & 0 & 0 & \\
\vdots & & & & & & & & \ddots
\end{array}\right)
$$

The following lemma will be crucial.

Lemma 4. Fix $k \geq 1$ and let $\mathbf{P}_{k}$ denote the $2^{k} \times 2^{k}$ upper left submatrix of $\mathbf{P}$. Then the sum modulo 2 of row $i$ in $\mathbf{P}_{k}$ is 0 for $0 \leq i \leq 2^{k}-1$ and is 1 for $i=2^{k}-1$.

First Proof The sum of row $i$ in $\mathbf{P}_{k}$ is, modulo 2,

$$
\sum_{j=0}^{2^{k}-1} p_{i j}=\sum_{j=0}^{2^{k}-1}\left(\begin{array}{c}
i+j \\
i
\end{array}\right)=\left(\begin{array}{c}
i+2^{k} \\
i+1
\end{array}\right)=\left(\begin{array}{c}
i+2^{k} \\
i+1,2^{k}-1
\end{array}\right)
$$

and for $i<2^{k}-1$ there is clearly at least one carry in the addition of $i+1$ and $2^{k}-1$ in base 2 so that, by Kummer's well known criterion, $2 \mid\left(\begin{array}{c}i+2^{k} \\ i+1,2^{k}-1\end{array}\right)$ and the sum of row $i$ is 0 $(\bmod 2)$. On the other hand, for $i=2^{k}-1$ there are no carries, so $2 \nmid\left(\begin{array}{c}i+2^{k} \\ i+1,2^{k}-1\end{array}\right)$ and the sum of row $i$ is $1(\bmod 2)$.

Second Proof The matrix $\mathbf{P}_{k}$ is the $2 \times 2$ block matrix

$$
\left(\begin{array}{cc}
P_{k-1} & P_{k-1} \\
P_{k-1} & 0
\end{array}\right)
$$

for $k \geq 2$, and the lemma follows immediately by induction.

Now let $p(\mathbf{c})$ denote the mod-2 parity of a composition $\mathbf{c}: p(\mathbf{c})=1$ if $\# \mathbf{c}$ is odd, $=0$ if $\# \mathbf{c}$ is even. For purposes of addition mod 2, represent the augmentation operators $\mathrm{P}$ and 
I by 0 and 1 respectively so that, for example, $p(\mathrm{~A}(\mathbf{c}))=p(\mathbf{c})+\mathrm{A}+1$ for $\mathrm{A}=\mathrm{P}$ or I by Lemma 2. Then the parity of $\mathbf{d}_{i+1}$ above can be obtained from the following commutative diagram (all addition modulo 2 )



The parity vector for a list of compositions $\left(\mathbf{c}_{i}\right)_{i=1}^{m}$ is $\left(p\left(\mathbf{c}_{i}\right)\right)_{i=1}^{m}$. This leads to

Lemma 5. Let $\mathbf{p}$ denote the parity vector for the $F$-orbit $\left(\mathbf{c}_{i}\right)_{i=1}^{m}$ of the composition $\mathbf{c}_{1}$. Then the parity vector for $B\left(\mathbf{c}_{1}, \mathrm{~A}\right)$ is

$$
\mathrm{Sp}+\mathrm{Se}_{m}+(\mathrm{A}+1) \mathbf{e}_{m} .
$$

Now we are ready to prove the main result of this section concerning the orbits of $F$ on primitive $D U U$-avoiding Dyck $n$-paths identified with the set $\mathcal{C}_{n}$ of compositions of $n$ that end with a 1 . The parity of an orbit is the sum mod 2 of the parities of the compositions comprising the orbit, in other words, the parity of the total number of entries in all the compositions.

Theorem 6. For each $n \geq 1$,

(i) all $F$-orbits on $\mathcal{C}_{n}$ have the same length and this length is a power of 2 , and

(ii) all $F$-orbits on $\mathcal{C}_{n}$ have the same parity.

The powers in $(i)$ and the parities in (ii) are given as follows:

The power (i.e. the exponent) is 0 for $n=1$ and increases monotonically with $n$, staying constant except for a jump of 1 when $n$ is of the form $2^{k}+2, k \geq 0$. The parity is 1 if $n$ is 1 or has the form $2^{k}+1, k \geq 1$, and is 0 otherwise.

\begin{tabular}{c|cccccccccccccccccc}
$n$ & 1 & 2 & 3 & 4 & 5 & 6 & 7 & 8 & 9 & 10 & 11 & 12 & 13 & 14 & 15 & 16 & 17 & 18 \\
\hline power & 0 & 0 & 1 & 2 & 2 & 3 & 3 & 3 & 3 & 4 & 4 & 4 & 4 & 4 & 4 & 4 & 4 & 5 \\
parity & 1 & 0 & 1 & 0 & 1 & 0 & 0 & 0 & 1 & 0 & 0 & 0 & 0 & 0 & 0 & 0 & 1 & 0
\end{tabular}

Proof We consider orbits generated by the augmentation operators $\mathrm{P}$ and $\mathrm{I}$ and proceed by induction on $n$. No orbits are missed because all compositions, in particular those ending 1 , can be generated from the unique composition of 1 by successive application of $\mathrm{P}$ and $\mathrm{I}$. The base cases $n=1,2,3$ are clear from the orbits $(1) \rightarrow(1),(1,1) \rightarrow(1,1),(2,1) \rightarrow(1,1,1) \rightarrow(2,1)$. To establish the induction step, suppose given an orbit, $\operatorname{orb}(\mathbf{c})$, in $\mathcal{C}_{2^{k}+1}(k \geq 1)$ of length $2^{k}$ with parity vector $\mathbf{p}$ and (total) parity 1 . Then, using Lemma 5 twice, the next orbit $B(\mathbf{c}, \mathrm{P}, \mathrm{I})$ has parity vector

$$
\mathbf{p}_{1}=\left(\mathbf{S} \mathbf{p}, \mathbf{S p}+\mathbf{e}_{2^{k}}\right)+\mathbf{S e}_{2^{k+1}}
$$


with parity (Sp's cancel out) $\underbrace{1+1+\ldots+1}_{2^{k}}+\underbrace{1+0+1+0+\ldots+1+0}_{2^{k+1}}=0$ for $k \geq 1$. Successively "bump up" this orbit using $A=\epsilon_{1}, \epsilon_{2}, \ldots$, in turn until the parity hits 1 again. With $\operatorname{Sum}(\mathbf{v})$ denoting the sum of the entries in $\mathbf{v}$, the successive parity vectors $\mathbf{p}_{1}, \mathbf{p}_{2}, \ldots$ are given by

$$
\begin{aligned}
\mathbf{p}_{i}=\left(\mathrm{S}^{i} \mathbf{p}, \mathrm{S}^{i} \mathbf{p}+\sum_{j=1}^{i-2} \operatorname{Sum}\left(\mathrm{S}^{j} \mathbf{p}\right) \mathrm{S}^{i-1-j} \mathbf{e}_{2^{k}}+\mathrm{S}^{i-1} \mathbf{e}_{2^{k}}\right)+ \\
\mathrm{S}^{i} \mathbf{e}_{2^{k+1}}+\mathrm{S}^{i-1} \mathbf{e}_{2^{k+1}}+\sum_{j=1}^{i-2} \epsilon_{j} \mathrm{~S}^{i-1-j} \mathbf{e}_{2^{k+1}}+\left(\epsilon_{i-1}+1\right) \mathbf{e}_{2^{k+1}} .
\end{aligned}
$$

Applying Lemma 4 we see that, independent of the $\epsilon_{i}{ }^{\prime}$ s, $\mathbf{p}_{i}$ has sum 0 for $i<2^{k}-1$ and sum 1 for $i=2^{k}-1$. This establishes the induction step in the theorem.

Corollary 7. For $n \geq 3$ the length of each F-orbit in $\mathcal{P}_{n}(D U U)$ is $2^{k}$, where $k$ is the number of bits in the base-2 expansion of $n-2$. (For $n=1$ or $2,\left|\mathcal{P}_{n}(D U U)\right|=1$.)

Proof This is just a restatement of part of the preceding Theorem.

\section{$5 \quad F$ on Primitive $D U U$-containing Paths}

The preceding section analyzed $F$ on $\mathcal{P}(D U U)$, primitive Dyck paths avoiding $D U U$. Now we consider $F$ on $\mathcal{P}[D U U]$, the primitive Dyck paths containing a $D U U$. Every $P \in \mathcal{P}[D U U]$ has the form $A Q B$ where

(i) $A$ consists of one or more $U_{\mathrm{s}}$

(ii) The concatenation $A B \in \mathcal{P}(D U U)$

(iii) $Q$ is a nonempty Dyck path, $Q \notin \mathcal{P}$ and $Q$ ends with $D D$ (and hence $Q$ contains a $D U U$ at its ground level).

To see this, locate the rightmost of the lowest $D U U \mathrm{~s}$ in $P$, say at height $h$. Then $A=U^{h}, Q$ starts at step number $h+1$ and extends through the matching downstep of the middle $U$ in this rightmost lowest $D U U$, and $B$ consists of the rest of the path.

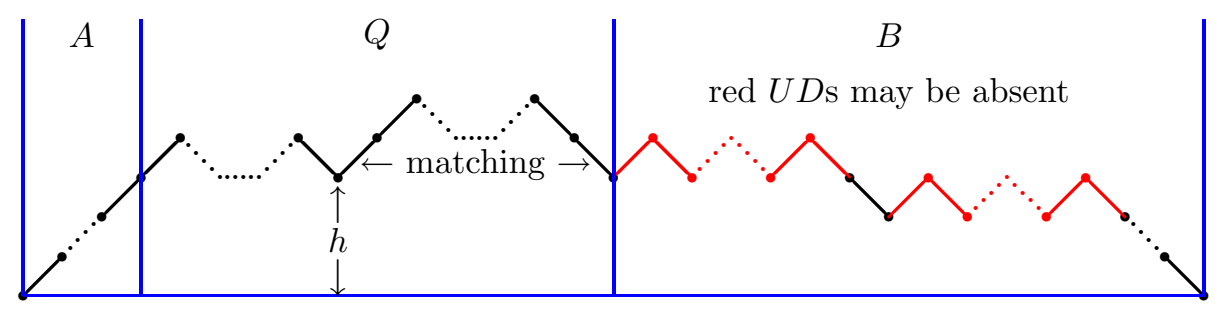

The $A Q B$ decomposition of a path containing a $D U U$ 
Call the path $A B$ the (DUU-avoiding) skeleton of $P$ and $Q$ the (DUU-containing) body of $P$. In case $P \in \mathcal{P}(D U U)$, its skeleton is itself and its body is empty. If the skeleton of $P$ is $U D$, then $P$ is uniquely determined by its skeleton and body. On the other hand, a skeleton of size $\geq 2$ and a nonempty body determine precisely two paths $P$ in $\mathcal{P}[D U U]$, obtained by inserting the body at either the top or the bottom of the first peak upstep in the skeleton, as illustrated.

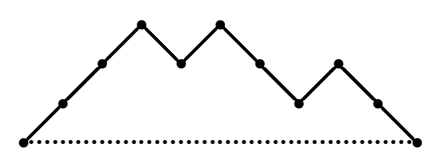

skeleton $S$
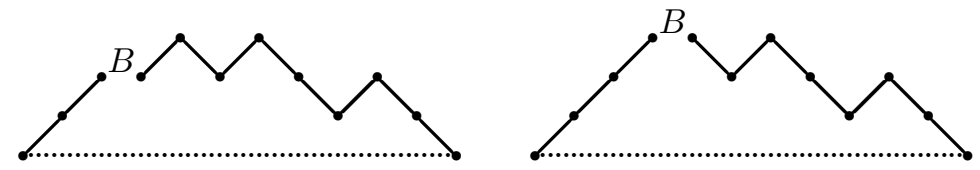

two possible $P \mathrm{~s}$

Recapturing a path $P \in \mathcal{P}[D U U]$ from a skeleton $S$ and body $B$

Thus paths in $\mathcal{P}[D U U]$ correspond bijectively to triples $(S, B$, pos $)$ where $S \in \mathcal{P}(D U U)$ is the skeleton, $B \neq \epsilon$ is the body, and pos =top or bot according as $B$ is positioned at the top or bottom of the first peak upstep in $S$, with the proviso that pos $=$ top if $S=U D$. For pos $\in\{$ top, bot $\}$, pos' denotes the other one.

In these terms, $F$ can be specified recursively on $\mathcal{P}[D U U]$ as follows.

\section{Proposition 8.}

$$
F((S, B, p o s))=\left\{\begin{array}{l}
(F(S), F(B), \text { pos }) \text { if height }(S) \text { is odd, and } \\
\left(F(S), F(B), \text { pos }^{\prime}\right) \text { if height }(S) \text { is even. }
\end{array}\right.
$$

Proof For a Dyck path $P$ containing a $D U U$, let $h(P)$ denote the height of the lowest valley vertex occurring in a $D U U$. Thus, if $P$ ends with $D D$, then $h(P)=0 \Leftrightarrow P$ is not primitive. Now suppose $P \in \mathcal{P}[D U U]$. The result holds for $h(P)=1$ (second case in Diagram 1 of $\S 3$ ). If $h(P) \geq 2$ (first case in Diagram 1), then $P$ has the form $U^{2} P^{\prime}(U D)^{a} D(U D)^{b} D$ with $a, b \geq 0$ and $P^{\prime}$ a Dyck path that ends with $D D$. So $F(P)=$ $U^{b+1} F\left(P^{\prime}\right)(U D)^{a+1} D^{b+1}$ and $h\left(P^{\prime}\right)=h(P)-2$. These two facts are the basis for a proof by induction that begins as follows. If $h\left(P^{\prime}\right)=0$, then the body of $F(P)$ has position = bottom, while the body of $P$ has position bottom or top according as $a \geq 1$ or $a=0$. In the former case, the skeleton of $P$ has height 3 and position has been preserved, while in the latter it has height 2 and position has been reversed.

\section{LCO Forests and the Orbits of $F$}

Iterating the skeleton-body-position decomposition of $\S 5$ on each component, a Dyck 
path has a forest representation as illustrated below. Each vertex represents a skeleton and is labeled with the corresponding composition. When needed, a color (top or bot) is also applied to a vertex to capture the position of that skeleton's body.
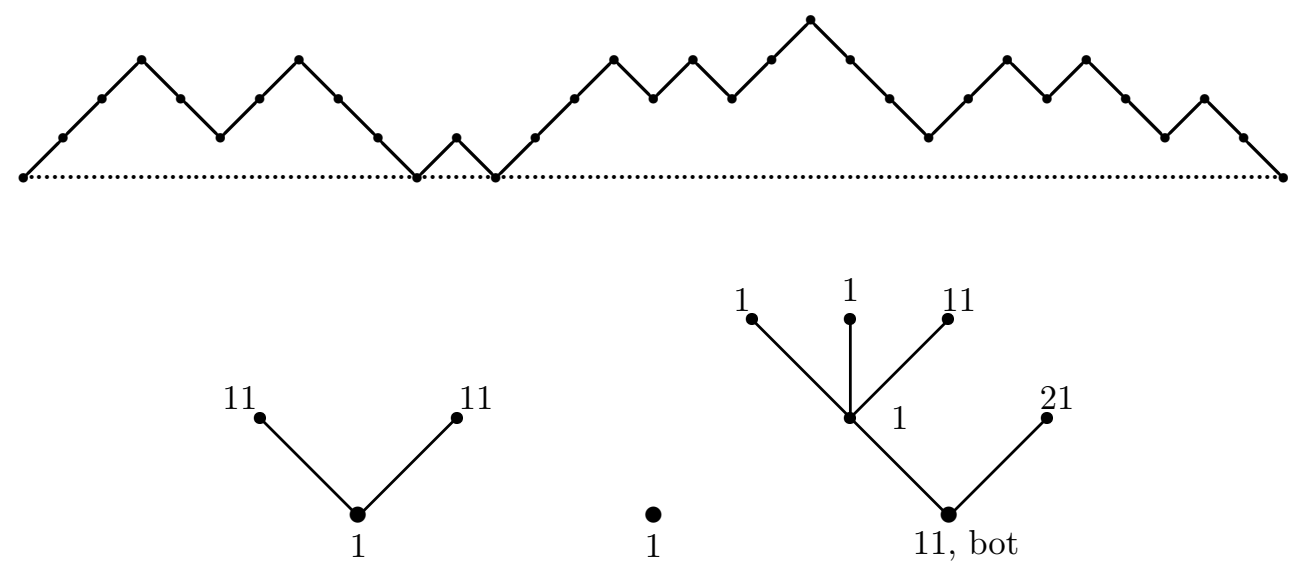

A Dyck path and corresponding LCO forest

The 3 trees in the forest correspond to the 3 components of the Dyck path. The skeleton of the first component is $U D$ and its body has 2 identical components, each consisting of a skeleton alone, yielding the leftmost tree. The skeleton of the third component is $U U D D$ and its body is positioned at the bottom of its first peak upstep, and so on. Call this forest the LCO (labeled, colored, ordered) forest corresponding to the Dyck path. Here is the precise definition.

Definition 9. An LCO forest is a labeled, colored, ordered forest such that

1. the underlying forest consists of a list of ordered trees (a tree may consist of a root only)

2. no vertex has outdegree 1 (i.e., exactly one child)

3. each vertex is labeled with a composition that ends with a 1

4. each vertex possessing children and labeled with a composition of size $\geq 2$ is also colored top or bot

5. each childless vertex that has a parent and is the rightmost child of its parent has label composition of size $\geq 2$.

The size of an LCO forest is the sum of the sizes of its label compositions. The correspondence Dyck path $\leftrightarrow$ LCO forest preserves size, and primitive Dyck paths correspond to one-tree forests. Thus we have

Proposition 10. The number of $L C O$ forests of size $n$ is the Catalan number $C_{n}$, as is the number of one-tree LCO forests of size $n+1$. 
The $C_{4}=14$ one-tree LCO forests corresponding to primitive Dyck 5-paths are shown, partitioned into $F$-orbits.

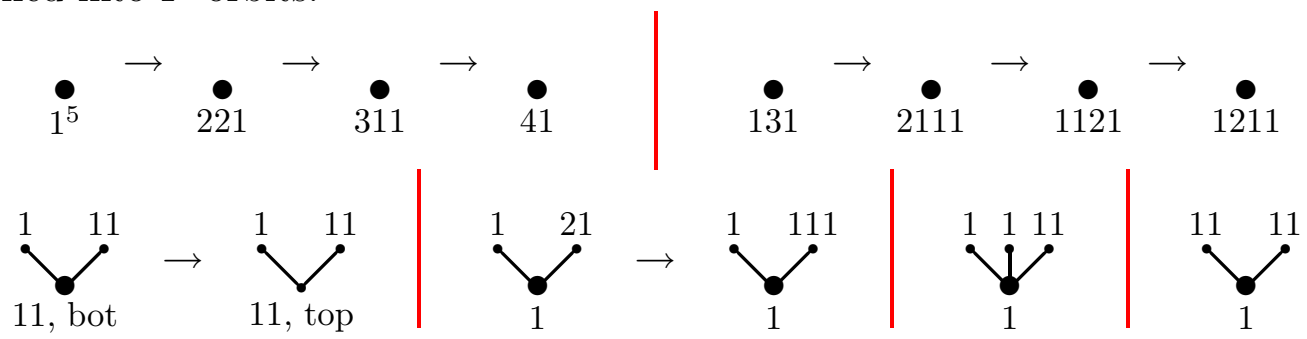

The LCO one-tree forests of size 5 , partitioned into $F$-orbits

We can now give an explicit description of $F$ on Dyck paths identified with LCO forests. By Proposition 8, $F$ acts as follows on an LCO forest:

- the underlying list of ordered trees is preserved

- each label c becomes $F(\mathbf{c})$ as defined in Prop. 1

- each color (top/bot) is preserved or switched according as the associated label c has odd or even length.

From this description and Corollary 7 , the size of the $F$-orbit of a Dyck path $P$ can be determined as in the following Proposition. In an LCO forest, call a childless vertex a leaf; in particular, an isolated root is a leaf. Recall that the labels in an LCO forest are compositions that end with a 1 , and the size of a composition is the sum of its entries.

Proposition 11. In the LCO forest for a Dyck path $P$, let $\ell$ denote the maximum size of a leaf label and $i$ the maximum size of a non-leaf label. Let $k$ denote the number of bits in the base-2 expansion of $\max \{\ell-2, i-1\}$ (with $k:=0$ if $\max \{\ell-2, i-1\} \leq 0)$. Then the F-orbit of $P$ has size $2^{k}$.

It is also possible to specify orbit sizes in terms of subpath avoidance. For Dyck paths $Q$ and $R$, let $Q$ top $R$ (resp. $Q$ bot $R$ ) denote the Dyck path obtained by inserting $R$ at the top (resp. bottom) of the first peak upstep in $Q$. Then the $F$-orbit of a Dyck path $P$ has size $\leq 2^{k}$ iff $P$ avoids subpaths in the set $\{Q$ top $R, Q$ bot $R: R \neq \epsilon, Q \in$ $\left.\mathcal{P}_{i}(D U U), 2^{k-1}+1<i \leq 2^{k}+1\right\}$. For $k \geq 1$, listing these $Q$ s explicitly would give $2^{2^{k}}-2^{2^{k-1}}$ proscribed patterns of the form $Q$ top $R, R \neq \epsilon$ (and the same number of the form $Q$ bot $R$ ). For $k=0$, that is, for fixed points of $F$, the proscribed patterns are $U P^{+} U D D$ and $U U P^{+} D D$ with $P^{+}$a nonempty Dyck path, and avoiding the first of these amounts to avoiding the subpath $D U D D$.

\section{Generating Functions}

The preceding section concludes with a negative characterization of Dyck paths that lie in an $F$-orbit of size $\leq 2^{k}$ - they must avoid certain subpaths. More useful for finding 
generating functions is a positive (and recursive) characterization for primitive Dyck paths in terms of the skeleton-body decomposition. Recall that the body of a Dyck path is either empty or a non-primitive Dyck path that ends with $D D$. Consider primitive Dyck paths that lie in an orbit of size $\leq 2^{k}$. For $k=0$ (fixed points), these paths are $U D, U U D D$ and $U R D$ where $R$ is a non-primitive Dyck path that ends with $D D$ and is a fixed point for $F$; in other words, primitive Dyck paths whose skeleton is of size $\leq 2$ and whose body is empty, together with primitive paths that have a skeleton of size 1 and a nonempty body that is a fixed point for $F$. In general, for $k \geq 0$, the primitive Dyck paths that lie in an orbit of size $\leq 2^{k}$ consist of two disjoint classes: (i) primitive Dyck paths whose skeleton is of size $\leq 2^{k}+1$ and whose body is empty, (ii) primitive Dyck paths that have a skeleton of size $\leq 2^{k}$ and a nonempty body lying in an orbit of size $\leq 2^{k}$.

From this characterization we can derive the generating function for the number of Dyck paths lying in an orbit of size $\leq 2^{k}$ as follows. Let $F_{k}(x), G_{k}(x), H_{k}(x)$ denote the respective generating functions for general Dyck paths, primitive Dyck paths, and non-primitive Dyck paths that end with $D D$ ( $x$ always marking size). We find

$$
\begin{aligned}
& F_{k}(x)=\underbrace{1}_{\substack{\text { empty } \\
\text { path }}}+\underbrace{G_{k}(x)}_{\begin{array}{c}
\text { primitive } \\
\text { path }
\end{array}} \underbrace{F_{k}(x)}_{\begin{array}{c}
\text { arbitrary } \\
\text { path }
\end{array}}, \\
& G_{k}(x)=\underbrace{x+x^{2}+2 x^{3}+4 x^{4}+\ldots+2^{2^{k}-1} x^{2^{k}+1}}_{\text {skeleton size } \leq 2^{k}+1, \text { body empty }}+ \\
& \underbrace{H_{k}(x)}_{\begin{array}{c}
\text { nonempty } \\
\text { body }
\end{array}}(\underbrace{x}_{\begin{array}{c}
\text { skeleton } \\
\text { size } 1
\end{array}}+\underbrace{2}_{\begin{array}{c}
\text { place } \\
\text { body }
\end{array}}(\underbrace{x^{2}+2 x^{3}+4 x^{4}+\ldots+2^{2^{k}-2} x^{2^{k}}}_{2 \leq \text { skeleton size } \leq 2^{k}})), \\
& H_{k}(x)=\underbrace{\left(F_{k}(x)-1\right)}_{\begin{array}{c}
\text { nonempty } \\
\text { path }
\end{array}}(\underbrace{G_{k}(x)-x}_{\begin{array}{c}
\text { primitive, } \\
\text { ends with } D D
\end{array}})
\end{aligned}
$$

After eliminating $G_{k}$ and $H_{k}$ from this system of equations and simplifying, we obtain

$$
F_{k}(x)=\frac{1-a_{k}-\sqrt{1-4 x+\frac{a_{k}\left(2-a_{k}\right) x}{1-x}}}{2 x-a_{k}},
$$

where $a_{k}=(2 x)^{2^{k}+1}$. With $F_{k}$ in this form it is clear, as expected, that $\lim _{k \rightarrow \infty} F_{k}(x)=$ $\frac{1-\sqrt{1-4 x}}{2 x}$, the generating function for the Catalan numbers.

It follows that the generating function for the number of orbits of (exact) size $2^{k}$ is

$$
\begin{cases}F_{0}(x) & \text { if } k=0, \text { and } \\ \frac{F_{k}(x)-F_{k-1}(x)}{2^{k}} & \text { if } k \geq 1 .\end{cases}
$$

This yields Table 1 wherein a divisibility property is evident in the columns on the right. 


\begin{tabular}{c|cccccc}
$n \backslash k$ & 0 & 1 & 2 & 3 & 4 & 5 \\
\hline 1 & 1 & & & & & \\
2 & 2 & & & & & \\
3 & 3 & 1 & & & & \\
4 & 6 & 2 & 1 & & & \\
5 & 12 & 7 & 4 & & & \\
6 & 26 & 23 & 11 & 2 & & \\
7 & 59 & 71 & 41 & 8 & & \\
8 & 138 & 224 & 151 & 30 & & \\
9 & 332 & 709 & 550 & 114 & & \\
10 & 814 & 2253 & 1993 & 406 & 16 & \\
11 & $*$ & 7189 & 7211 & 1564 & 64 & \\
12 & $*$ & $*$ & 26221 & 6010 & 240 & \\
13 & $*$ & $*$ & 95583 & 23062 & 912 & \\
14 & $*$ & $*$ & $*$ & 88530 & 3504 & \\
15 & $*$ & $*$ & $*$ & $*$ & 13536 & \\
16 & $*$ & $*$ & $*$ & $*$ & 52432 & \\
17 & $*$ & $*$ & $*$ & $*$ & 203440 & \\
18 & $*$ & $*$ & $*$ & $*$ & 786320 & 2048
\end{tabular}

Table 1: Number of orbits of size $2^{k}$ of $F$ on Dyck $n$-paths

Proposition 12. On Dyckn-paths, the number of F-orbits of size $2^{k}$ is divisible by $2^{2^{k-1}-k}$ for all $n, k \geq 1$.

Proof The expression $\frac{F_{k}(x)-F_{k-1}(x)}{2^{k}}$ in (2) simplifies to

$$
\frac{(2 x-1) b-\sqrt{S_{1}}+(1+b) \sqrt{S_{2}}}{2^{k+1} x\left(1-b^{2}\right)}
$$

where $b=(2 x)^{2^{k-1}}, S_{1}=1-4 x+\frac{4 x^{2} b^{2}\left(1-b^{2} x\right)}{1-x}, S_{2}=1-4 x+\frac{4 x^{2} b(1-b x)}{1-x}$. The numerator in (3) vanishes for $b=0$. Hence its series expansion in $b$ has no constant term and so, in its series expansion in $x$ (with $b$ a fixed indeterminate), every coefficient is divisible by $b$. Every coefficient is also divisible by 2 because the numerator can be expressed

$$
(2 x-1) b-\sqrt{1-4 X}+(1+b) \sqrt{1-4 Y}
$$

where $X$ and $Y$ are integer-coefficient power series in $b$ and $x$. Since $\sqrt{1-4 Z}=1-$ $2 \sum_{n \geq 0} C_{n} Z^{n+1},(4)$ becomes

$$
(2 x-1) b-\left(1-2 \sum_{n \geq 0} C_{n} X^{n+1}\right)+(1+b)\left(1-2 \sum_{n \geq 0} C_{n} Y^{n+1}\right)
$$

which reduces to $0 \bmod 2$; hence the coefficients are also divisible by 2 . Divisibility by $2 b$ now implies that when $b$ is replaced by its actual value $(2 x)^{2^{k-1}}$ the expansion of the 
numerator in (3) as a power series in $x$ has every coefficient divisible by $(2)^{2^{k-1}+1}$. Taking account of the $2^{k+1}$ factor in the denominator (the " $x$ " cancels), the net result is that every coefficient in the series expansion of $\frac{F_{k}(x)-F_{k-1}(x)}{2^{k}}$ is divisible by $2^{2^{k-1}-k}$.

But more is true. The sequences obtained by dividing each column in Table 1 by its top entry approach a limit sequence $1,4,15,57, \ldots$. We can explain this combinatorially as follows. The first nonzero entry in column $k(k \geq 2)$ is in row $n=2^{k-1}+2$ and this entry is $2^{2^{k-1}-k}$ since the number of LCO forests of $2^{k-1}+2$ lying in an orbit of size $k$ is $2^{2^{k-1}}$. This is because Prop. 11 implies such forests are necessarily one-tree forests consisting only of a root labeled with $\mathbf{c} \in \mathcal{C}_{2^{k-1}+2}$ and $\left|\mathcal{C}_{n}\right|=2^{n-2}$. Fix $k \geq 1$ and let $\mathbf{c}_{i}$ denote a composition of size $2^{k-1}+2+i$. We will need to allow $i=-1$ but for $0 \leq i<2^{k-1}$, Prop. 11 implies $\mathbf{c}_{i}$ lies in an orbit of size $2^{k}$. Now for $i$ in this range, the criterion of Prop. 11 implies that the LCO forests of size $2^{k-1}+2+i$ lying in an orbit of size $2^{k}$ are precisely those satisfying the properties: (i) one label is $\mathbf{c}_{j}, j \geq-1$, (ii) if $j=-1$, then the vertex with label $\mathbf{c}_{j}$ has children, (iii) the sum of all label sizes is $2^{k-1}+2+i$. These forests can be represented as modified LCO forests.

Define a modified LCO forest to be an LCO forest whose composition labels include 11 (of size 2), one of which is circled, and with an additional integer label $j \geq-1$ on the vertex whose label is circled subject to the restriction $j \geq 0$ if this vertex is a leaf. The circled label represents the $\mathbf{c}_{j}$ label and is taken to be 11 simply to capture the fact that $\mathbf{c}_{j}$ has size $\geq 2$. Define the size of a modified LCO forest to be the sum of the non-circled labels plus $j$, and define its weight to be $2^{j}$.


A modified LCO forest of size $15-1=14$ and weight $\frac{1}{2}$

Because incrementing the subscript in $\mathbf{c}_{j}$ doubles the number of possibilities for the composition $\mathbf{c}_{j}$ and recalling that the number of possibilities for $\mathbf{c}_{j}$ with $j=0$ is $2^{2^{k-1}}$, we see that the number of orbits of size $2^{k}$ involving LCO forests of size $2^{k-1}+2+i$ $\left(0 \leq i<2^{k}\right)$ is $2^{2^{k-1}-k}$ times the total weight of all modified LCO forests of size $i$, and this total weight is independent of $k$.

The generating function for the total weight of modified LCO forests, with $x$ marking size, can be found by the recursion method used above to obtain the generating function $F_{k}(x)$ for Dyck paths counted by orbit size. It turns out to be

$$
\frac{1-2 x}{2 x^{3}}\left(\frac{1-3 x}{(1-x) \sqrt{1-4 x}}-1\right)
$$


with coefficient sequence $1,4,15,57,219,846,3277, \ldots$ (It can also be extracted analytically from $F_{k}(x)-F_{k-1}(x)$.)

We have shown that the sequence obtained from column $k$ agrees with the limit sequence in its first $2^{k-1}$ entries for $k \geq 1$, and thus the convergence is quite rapid.

Summing the columns in Table 1 above gives the total number of orbits of $F$ on Dyck n-paths, A127384 in OEIS.

\begin{tabular}{c|cccccccccc}
$n$ & 1 & 2 & 3 & 4 & 5 & 6 & 7 & 8 & 9 & 10 \\
\hline \# orbits & 1 & 2 & 4 & 9 & 23 & 62 & 179 & 543 & 1705 & 5482 \\
& & & & & &
\end{tabular}

The counting sequence for fixed points of $F$, with generating function $F_{0}(x)$, is sequence A086625.

\section{An Application}

Ordered trees and binary trees are manifestations of the Catalan numbers A000108. Donaghey and Shapiro [12] and Donaghey [13] list several types of restricted tree counted by the Motzkin numbers A001006. In particular, the following result is implicit in item III C of [13].

Proposition 13. The Motzkin number $M_{n}$ counts right-planted binary trees on $n+1$ edges with no erasable vertices.

Here, planted means the root has only one child, and erasable refers to a vertex incident with precisely 2 edges both of the same slope - the vertex could then be erased, preserving the slope, to produce a smaller binary tree. The $M_{3}=4$ such trees on 4 edges are shown.
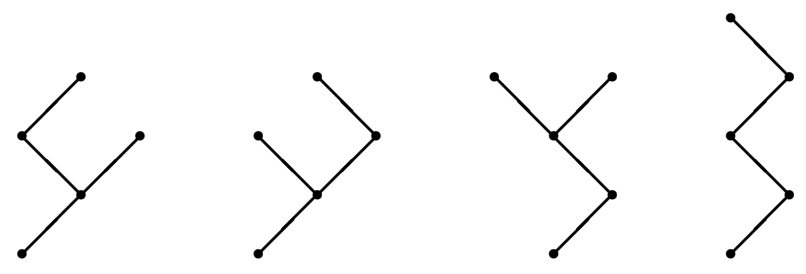

The right-planted binary 4-trees with no erasable vertices

Translating right-planted binary trees to Dyck paths using the bijections illustrated below (essentially Knuth's "natural correspondence" [14], due to Harary, Prins, and Tutte [15] and explicated by de Bruijn and Morselt [16]), Prop. 13 is equivalent to

Proposition 14. $M_{n}$ counts Dyck $(n+1)$-paths that end with $D D$ and avoid subpaths $D U D U$ and $U U P^{+} D D$ with $P^{+}$denoting a nonempty Dyck subpath. 




(1) rotate $45^{\circ}$

(2) swing down horizontal edges

(3) burrow up the edges and open out accordion-style

binary tree $\leftrightarrow$ Dyck path correspondence

We will use $F$ to give a bijective proof of Prop. 14 based on the fact [17] that $M_{n}$ also counts $D U D$-avoiding Dyck $(n+1)$-paths. (Of course, path reversal shows that \#UDUs and \# DUDs are equidistributed on Dyck paths.) Using the Iverson notation: [statement] $=1$ if statement is true, $=0$ if statement is false, define statistics $X$ and $Y$ on Dyck paths by $X=\# D U D \mathrm{~s}$ and $Y=\# D U D U \mathrm{~s}+\# U U P^{+} D D \mathrm{~s}+[$ paths ends with $U D]$ so that the paths in Prop. 14 are those with $Y=0$. Prop. 14 then follows from

Proposition 15. On Dyck n-paths with $n \geq 2, F$ sends the statistic $X$ to the statistic $Y$.

Proof Routine by induction from the recursive definition of $F$. However, using the explicit form of $F$, it is also possible to specify precisely which $D U D$ s correspond to each of the three summands in $Y$. For this purpose, given a $D U D$ in a Dyck path $P$, say $D_{1} U_{2} D_{3}$ (subscripts used simply to identify the individual steps), let $S\left(D_{1} U_{2} D_{3}\right)$ denote the longest Dyck subpath of $P$ containing $D_{1} U_{2} D_{3}$ in its skeleton and let $h$ denote the height at which $D_{1} U_{2} D_{3}$ terminates in $S\left(D_{1} U_{2} D_{3}\right)$. If $h$ is odd, $D_{1} U_{2} D_{3}$ is immediately followed in $P$ by $D_{4}$ or by $U D_{4}$ (it cannot be followed by $U U$ ). In either case, let $U_{4}$ be the matching upstep for $D_{4}$. Then the steps $D_{1}, U_{2}, D_{3}, U_{4}$ show up in $F(P)$ as part of a subpath $U_{4} U_{2} P^{+} D_{3} D_{4}$ with $P^{+}$a Dyck path that ends with $D_{1}$. On the other hand, if $h$ is even, $D_{1} U_{2} D_{3}$ either (i) ends the path (here $S\left(D_{1} U_{2} D_{3}\right)=P$ and $h=0$ ) or is immediately followed by (ii) $U_{4}$ or (iii) $D$. In case (iii), let $U_{4}$ be the matching upstep. Then $D_{1}, U_{2}, D_{3}, U_{4}$ show up in $F(P)$ as a subpath in that order (cases (ii) and (iii)) or $F(P)$ ends with $U_{2} D_{3}$ (case (i)). The details are left to the reader.

\section{Statistics Suggested by LCO Forests}

There are various natural statistics on LCO forests, some of which give interesting counting results. Here we present two such. First let us count one-tree LCO forests by size of root label. This is equivalent to counting primitive Dyck paths by skeleton size. Recall that the generalized Catalan number sequence $\left(C_{n}^{(j)}\right)_{n \geq 0}$ with $C_{n}^{(j)}:=\frac{j}{2 n+j}\left(\begin{array}{c}2 n+j \\ n\end{array}\right)$ is the $j$-fold convolution of the ordinary Catalan number sequence A000108. (See [18] for a 
nice bijective proof.) And, as noted above, in the skeleton-body-position decomposition of a primitive Dyck path, if the body is nonempty it contains a $D U U$ at (its own) ground level and ends with $D D$.

Lemma 16. The number of Dyck n-paths that contain a DUU at ground level and end with $D D$ is $C_{n-3}^{(4)}$.

Proof In such a path, let $U_{0}$ denote the middle $U$ of the last $D U U$ at ground level. The path then has the form $A U_{0} B D$ where $A$ and $B$ are arbitrary nonempty Dyck paths, counted by $C_{n-1}^{(2)}$. So the desired counting sequence is the convolution of $\left(C_{n-1}^{(2)}\right)$ with itself and, taking the $U_{0} D$ into account, the lemma follows.

The number of primitive $D U U$-avoiding Dyck $k$-paths is 1 if $k=1$, and $2^{k-2}$ if $k \geq 2$. But if $k \geq 2$, there are two choices (top/bottom) to insert the body. So the number of primitive Dyck $(n+1)$-paths with skeleton size $k$ is $2^{k-1} C_{n-k-2}^{(4)}$ for $1 \leq k \leq n-2$ and is $2^{n-1}$ for $k=n+1$. Since there are $C_{n}$ primitive Dyck $(n+1)$-paths altogether, counting by skeleton size yields a combinatorial interpretation of the following identity.

\section{Proposition 17.}

$$
C_{n}=2^{n-1}+\sum_{k=1}^{n-2} \frac{2^{k}}{n-k}\left(\begin{array}{c}
2 n-2 k \\
n-2-k
\end{array}\right)
$$

Recall that an isolated root in an LCO forest is considered a leaf. Counting by number of leaves yields a pretty generating function.

Proposition 18. The generating function for LCO forests by number of leaves ( $x$ marks size, $y$ marks number of leaves ) is

$$
\frac{1-\sqrt{1-4 x \frac{1-x}{1-x y}}}{2 x} .
$$

The first few values are given in Table 2.

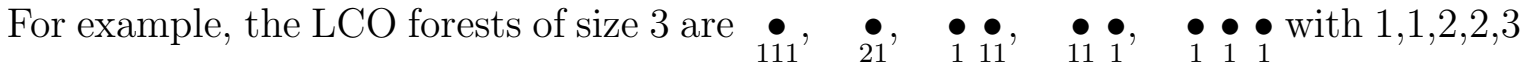
leaves respectively.

Proof Observe that deleting the root from a one-tree LCO forest leaves an LCO forest that is either (i) empty, or (ii) consists of at least 2 trees (condition 2 in Definition 9 of $\S 6)$ and if the last tree consists of a root only then its composition label has size $\geq 2$ (condition 5 ). So let $F(x, y)$ denote the desired generating function, $G(x, y)$ the generating function for 1-tree LCO forests, and $H(x, y)$ the generating function for the LCO forests satisfying condition (ii).

We need three equations to determine $F, G, H$ :

1. An LCO forest is either empty or consists of a 1-tree LCO forest followed by an arbitrary LCO forest. Hence

$$
F=1+G F
$$




\begin{tabular}{c|cccccccc}
$n \backslash k$ & 1 & 2 & 3 & 4 & 5 & 6 & 7 & 8 \\
\hline 1 & 1 & & & & & & & \\
2 & 1 & 1 & & & & & & \\
3 & 2 & 2 & 1 & & & & & \\
4 & 4 & 6 & 3 & 1 & & & & \\
5 & 8 & 17 & 12 & 4 & 1 & & & \\
6 & 16 & 46 & 44 & 20 & 5 & 1 & & \\
7 & 32 & 120 & 150 & 90 & 30 & 6 & 1 & \\
8 & 64 & 304 & 482 & 370 & 160 & 42 & 7 & 1
\end{tabular}

Table 2: number of LCO forests of size $n$ with $k$ leaves

2. Partition 1-tree LCO forests into four classes with respective contributions to $G$ as shown

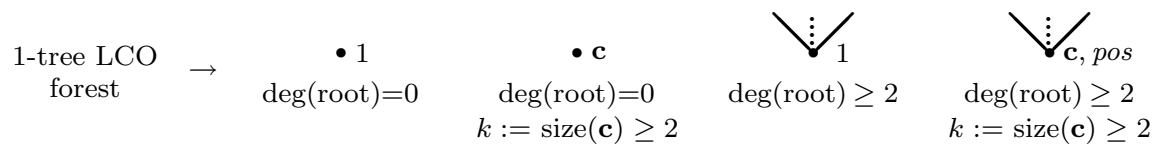

$$
\begin{aligned}
& \begin{array}{c}
\text { contribution } \\
\text { to } G
\end{array} \rightarrow \quad x y \quad 2^{k-2} x^{k} y \quad x H \quad 2^{k-2} 2 x^{k} H
\end{aligned}
$$

(Recall a label composition ends with a 1 and there are $2^{k-2}$ such of size $k$.) This yields

$$
G=x y+\frac{x^{2} y}{1-2 x}+\frac{x H}{1-2 x}
$$

3. An LCO forest counted by $H$ consists of a nonempty LCO forest followed by a 1tree LCO forest such that the tree does not consist of a single vertex labeled 1 (this vertex would be a root and a leaf). Hence

$$
H=(F-1)(G-x y)
$$

Eliminating $G$ and $H$ from these three equations yields a quadratic for $F$ from which the Proposition follows.

Acknowledgments I am very grateful to Emeric Deutsch for a careful reading of the paper and many helpful suggestions, including the second proof of Lemma 4.

\section{References}

[1] David Callan, Two bijections for Dyck path parameters, math.CO/0406381, 2004, 4pp.

[2] David Callan, Some bijections and identities for the Catalan and Fine numbers, Sém. Lothar. Combin. 53 (2004/06), Art. B53e, 16 pp. 
[3] Emeric Deutsch, An involution on Dyck paths and its consequences. Discrete Math. 204 (1999), no. 1-3, 163-166.

[4] Emeric Deutsch, A bijection on Dyck paths and its consequences, Discrete Math. 179 (1998), no. 1-3, 253-256.

[5] Emeric Deutsch, A bijection on ordered trees and its consequences, J. Combin. Theory Ser. A 90 (2000), no. 1, 210-215.

[6] Emeric Deutsch and Sergi Elizalde, A simple and unusual bijection for Dyck paths and its consequences, Ann. Comb. 7 (2003), no. 3, 281-297.

[7] Robert Donaghey, Automorphisms on Catalan trees and bracketings. J. Combinatorial Theory Ser. B 29 (1980), no. 1, 75-90. MR0584162

[8] Donald Knuth, Art of Computer Programming, Vol.4, Fascicle 4: Generating all Trees - History of Combinatorial Generation, Addison-Wesley, 2006, vi+120pp, draft available from http://www-cs-faculty.stanford.edu/ knuth/fasc4a.ps.gz

[9] J.-C. Lalanne, Une involution sur les chemins de Dyck, European J. Combin. 13 (1992), no. 6, 477-487.

[10] J.-C. Lalanne, Sur une involution sur les chemins de Dyck, Conference on Formal Power Series and Algebraic Combinatorics Theoret. Comput. Sci. 117 (1993), no. 1-2, 203-215.

[11] J. Vaillé, Une bijection explicative de plusieurs propriétés remarquables des ponts, European J. Combin. 18 (1997), no. 1, 117-124.

[12] Robert Donaghey and Louis Shapiro, Motzkin numbers, J. Combinatorial Theory Ser. A 23, 291-301, 1977. MR0505544

[13] Robert Donaghey, Restricted plane tree representations of four Motzkin-Catalan equations, J. Combinatorial Theory Ser. B 22, (1977), no. 2, 114-121, 1977. MR0432532

[14] D. Knuth, The Art of Computer Programming, Sorting and Searching, Vol. 1, 3rd ed., Addison-Wesley Professional, Boston, MA, 1997.

[15] Frank Harary, Geert Prins, and W. T. Tutte, The number of plane trees, Nederl. Akad. Wetensch. Proc. Ser. A 67 = Indag. Math. 26 (1964), 319-329.

[16] N. G. de Bruijn and B. J. M. Morselt, A note on plane trees, J. Combinatorial Theory 2, (1967), 27-34.

[17] Y. Sun, The statistic "number of udu's" in Dyck paths, Disc. Math., 287 (2004), Issue 1-3 (October 2004), 177-186.

[18] Wen-jin Woan, Uniform partitions of lattice paths and Chung-Feller generalizations. Amer. Math. Monthly 108 (2001), no. 6, 556-559. 